\title{
Decreased parahippocampal activity in associative priming: Evidence from an event-related fMRI study
}

\author{
jiongjiong Yang, ${ }^{1,4}$ Axel Meckingler, ${ }^{2}$ Mingwei Xu, ${ }^{3}$ Yanbing Zhao, ${ }^{1}$ \\ and Xuchu Weng ${ }^{3}$ \\ ${ }^{1}$ Department of Psychology, Peking University, Beijing 100871, China; ${ }^{2}$ Experimental Neuropsychology Unit, \\ Department of Psychology, Saarland University, D-66123 Saarbruecken, Germany; ${ }^{3}$ Laboratory of Higher Brain Functions, \\ Institute of Psychology, Chinese Academy of Sciences, Beijing 100012, China
}

\begin{abstract}
In recent years, there has been intense debate on the neural basis of associative priming, particularly on the role of the medial temporal lobe (MTL) in retrieving associative information without awareness. In this study, event-related fMRI was used while healthy subjects performed a perceptual identification task on briefly presented unrelated word pairs and an associative recognition memory task. Contamination of priming by explicit memory was successfully controlled, as associative priming and explicit memory were dissociated on the behavioral level. The fMRI results showed a functional dissociation within the MTL with respect to associative priming effects. The right parahippocampal cortex, but not the hippocampus, showed decreased activation for old vs. new pairs and old vs. recombined pairs (associative priming). The bilateral hippocampus and the right parahippocampal cortex were involved in explicit associative memory. These data provide evidence that subregions of the MTL participate in associative priming even when explicit involvement was controlled. Thus, different regions within the MTL play distinct roles in explicit and implicit associative memory.
\end{abstract}

Priming effects can be classified by changes of a person's implicit memory upon individual targets (item priming) or upon associations between two or more targets (e.g., window-reason, associative priming). Converging evidence from neuropsychological and neuroimaging studies have suggested that the extrastriate cortex and the prefrontal cortex are involved in item priming, showing decreased activation for old items compared with new items (for reviews, see Henson 2003; Schacter et al. 2004; Martin and Gotts 2005). In contrast, the neural basis of associative priming remains unclear. An important issue is whether the medial temporal lobe (MTL) is involved in retrieving associative information without awareness. The findings on associative priming in amnesic patients are conflicting. Some studies have shown that amnesic patients are impaired in both associative priming and explicit memory, indicating that the MTL is involved in associative priming (e.g., Graf and Schacter 1985; Mayes and Gooding 1989; Shimamura and Squire 1989; Chun and Phelps 1999; Yang et al. 2003; Verfaellie et al. 2006). Other studies have shown that amnesic patients are impaired in explicit memory tasks but show normal performance in associative priming and item priming tasks, suggesting that the MTL does not appear to be essential for associative implicit memory (e.g., Moscovitch et al. 1986; Musen and Squire 1993; Gabrieli et al. 1997; Goshen-Gottstein et al. 2000; Verfaellie et al. 2006).

Despite the conflicting findings, the literature on amnesia suggests that associative priming is modulated by task demands, as it is less impaired in perceptual tasks but severely impaired in conceptual tasks. For example, when amnesic patients perform a lexical decision task for word pairs (e.g., Goshen-Gottstein et al. 2000), their reaction times (RT) were faster for old pairs than recombined pairs, and the performance was comparable to that of normal controls. In contrast, when amnesic patients performed conceptual tasks, such as stem-completion tasks (e.g.,

${ }^{4}$ Corresponding author.

E-mail yangjj@pku.edu.cn; fax 86-10-62761081.

Article is online at http://www.learnmem.org/cgi/doi/10.1101//m.900108.
Graf and Schacter 1985; Shimamura and Squire 1989) or crossmodal tasks (e.g., Badgaiyan et al. 1999), the accuracy for old pairs and recombined pairs or for items from the same and from different modalities did not differ. By this, their performance was clearly different from normal controls. It should be noted that when conceptual tasks require subjects to judge the configuration of the presented pairs (e.g., a related judgment task) (Verfaellie et al. 2006), amnesic patients manifest longer latencies for old rather than for recombined pairs, as normal subjects do. Also, when tasks require subjects to unitize the items into a whole, associative memory is preserved in amnesia (Mayes et al. 2004). Thus, it seems that the nature of association is an important factor for associative priming.

The inferences drawn from studies with amnesic patients are also complicated by uncontrollable factors concerning the location and the extent of MTL lesions due to clinical diversities. As indicated by recent studies, subregions of the MTL, e.g., the hippocampus and the parahippocampal gyrus (PHG), may play differential roles in memory processes (for reviews, see Aggleton and Brown 2006; Davachi 2006; Diana et al. 2007; Eichenbaum et al. 2007; Mayes et al. 2007; but also, see Squire et al. 2004). Specifically, the anterior PHG (i.e., entorhinal/perirhinal cortex) is more involved in familiarity-based remembering and the retrieval of unitized association, whereas the hippocampus is more involved in recollection-based remembering and the processing of arbitrary associations (Henson 2005; Diana et al. 2007). The posterior PHG seems to play a role in the retrieval of contextual information (Diana et al. 2007; Eichenbaum et al. 2007). Studies with amnesic patients suggested that MTL subregions other than the hippocampus are more related to implicit contextual task (Chun and Phelps 1999; Manns and Squire 2001; Verfaellie et al. 2006). For example, Chun and Phelps (1999) report that a group of amnesic patients had deficits in an implicit contextual learning task. However, if the damage was confined to the hippocampus, the patients' performance was preserved in the same task (Manns and Squire 2001). In addition to the implicit contextual task, patient studies that used perceptual identification tasks (e.g., 
Gabrieli et al. 1997; Yang et al. 2003) also obtained inconsistent findings. Gabrieli et al. (1997) found that amnesic patients identify old pairs better than recombined ones in amnesia, whereas Yang et al. (2003) found impaired associative priming. Note that most amnesic patients in the Gabrieli et al. (1997) study had lesions in the diencephalon and in the temporal lobes due to Korsakoff's disease (Gazzaniga et al. 2002), and the patients in the Yang et al. (2003) study mostly had lesions that were restricted to the MTL. These disagreements on specific tasks suggest that the heterogeneity in the location and the extent of MTL lesions may limit strong conclusions from patient studies on the brain regions involved in associative priming. Therefore, it merits further exploration whether different subregions of the MTLthe hippocampus and subregions of the $\mathrm{PHG}$ - contribute to associative priming.

Moreover, although recent neuroimaging studies have found that the MTL is activated in memory tasks for old items vs. recombined/control items (e.g., Badgaiyan et al. 2003; Henke et al. 2003a,b), its activation has been questioned by possible contamination of explicit memory (for review, see Schacter et al. 2004). As explicit memory tasks usually elicit increased activation in the brain (for review, see Buckner and Wheeler 2001) and as there is no distinct behavioral dissociation between explicit and implicit memory performance, the increased activation in the MTL is often considered to be a correlate of explicit memory (Squire et al. 1992; Badgaiyan et al. 1999, 2001, 2003; Schacter et al. 2004). For example, in a recent study by Henke et al. (2003a) that used both implicit and explicit retrieval conditions, normal subjects named the professions (e.g., artist, academic) of faces faster when they judged the profession categories correctly (i.e., the profession of a face during the encoding). Thus, it is difficult to exclude an involvement of explicit retrieval in implicit memory tasks. Given this, the control of explicit contamination is a crucial factor in exploring neural mechanisms of associative priming.

To address these issues and to further determine the role of the MTL in associative priming, two approaches were adopted in this study. First, fMRI was used to take advantage of its higher spatial resolution compared with that of the PET technique (Badgaiyan et al. 2003). It allows a fine-grained analysis of the MTL subregions contributing to associative memory tasks, which is difficult to accomplish in studies with amnesic patients. Second, a possible contribution of explicit memory to associative priming was controlled by employing a perceptual encoding task with Chinese words, in combination with a perceptual identification test. Perceptually based associative priming can be found in normal subjects (Goshen-Gottstein and Moscovitch 1995; Yang et al. 1999) and usually is preserved in amnesic patients (e.g., Moscovitch et al. 1986; Goshen-Gottstein et al. 2000). One advantage of using Chinese words (two characters) is that each Chinese character has a distinctive perceptual configuration, e.g., left-right (杯, cup), up-down (花, flower). Thus, subjects can judge the combination of the configurations and form perceptual associations between words (e.g., judge whether the two words had the same combined configurations) (Yang et al. 1999). In addition, by using a perceptual encoding task, subsequent explicit memory performance was at chance level, and accordingly, the possible explicit contamination of associative priming was excluded. Contributions from explicit memory were further controlled by the use of a perceptual identification task (Gabrieli et al. 1997; Yang et al. 2003), in which word pairs were quickly flashed with individually adjusted exposure durations. These manipulations allowed us to investigate the role of the subregions of the MTL in associative priming with a high control of contamination from explicit memory processes. We predicted that within the MTL, the PHG, but not the hippocampus, would be involved in the associative priming effect, whereas both the PHG and the hippocampus would be involved in associative recognition.

\section{Results}

\section{Behavioral results}

In this study, normal participants performed three different tasks (Fig. 1; Yang et al. 2003), i.e., a perceptual associative task (encoding task), a perceptual identification task (priming task), and an associative recognition task. Unrelated Chinese concrete word pairs were used as stimuli, which were divided into subsets of old, recombined, and new (only in the priming task) pairs. The behavioral data of the encoding and recognition tasks were collected from subjects in the fMRI and the behavioral group; as the results for the two groups were similar, only the behavioral group's data are reported in the text. As subjects identified the word pairs covertly in the scanner in the priming task, the behavioral performance for this task was collected from subjects in the behavioral group.

The behavioral results showed dissociation between implicit memory (item priming and associative priming) and explicit memory. Subjects identified old pairs more accurately than recombined pairs in the perceptual identification task, although they could not distinguish old pairs from recombined pairs in the explicit recognition memory task. In the perceptual identification task, subjects showed significant associative priming and item priming effects (Table 1 ). The main effect of word pair was significant, $F_{(2,34)}=31.62, P<0.0001$ for accuracy, and $F_{(2,14)}=5.03, P<0.02$ for RT. The old pairs were identified more accurately than the recombined pairs $\left(0.67\right.$ vs. $0.61, t_{(17)}=2.17$, $P<0.05)$, with no significant difference in $\mathrm{RT}\left(t_{(7)}=0.13\right.$, $P<0.9)$. In addition, the recombined pairs were identified more accurately $\left(0.61\right.$ vs. $\left.0.38, t_{(17)}=5.04, P<0.001\right)$ and faster $(863$ msec vs. $\left.964 \mathrm{msec}, t_{(7)}=2.6, P<0.05\right)$ than the new pairs.

On the other hand, their associative recognition performance (accuracy and RT) was at chance level, suggesting that they could not distinguish old pairs from recombined pairs. There was no significant difference between old and recombined
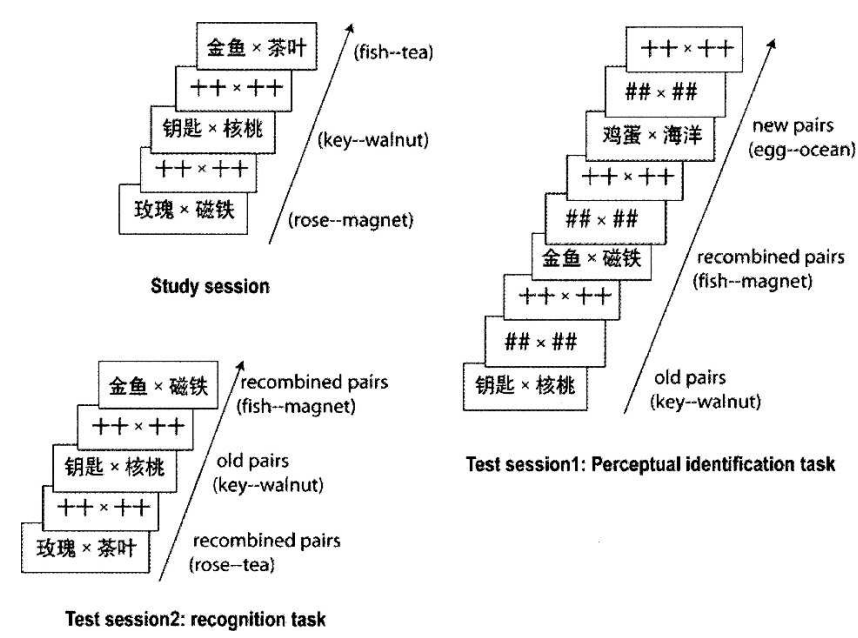

Test session1: Perceptual identification task

Figure 1. Illustration of the study and test sessions. In the study session, Chinese unrelated word pairs were presented. Subjects made a perceptually associative judgment (whether the two words had the same structure combination). During the perceptual identification task, three types of unrelated word pairs (old, recombined, and new) were presented with the predetermined duration in the intermixed order. Backward masking was used to minimize the explicit contamination. During the recognition task, word and recombined word pairs were presented, and subjects made an old/recombined judgment. 
Table 1. Behavioral data for the perceptual identification task and the associative recognition task (M \pm SD)

\begin{tabular}{|c|c|c|c|c|c|c|c|}
\hline & \multirow[b]{2}{*}{ Task } & \multicolumn{3}{|c|}{ Accuracy } & \multicolumn{3}{|c|}{ RT (msec) } \\
\hline & & Old & Recombined & New & Old & Recombined & New \\
\hline $\begin{array}{l}\text { Behavioral group } \\
\text { fMRI group }\end{array}$ & $\begin{array}{l}\text { Perceptual identification } \\
\text { Recognition } \\
\text { Recognition }\end{array}$ & $\begin{array}{l}0.67 \pm 0.22 \\
0.51 \pm 0.20 \\
0.43 \pm 0.25\end{array}$ & $\begin{array}{l}0.61 \pm 0.21 \\
0.43 \pm 0.27 \\
0.47 \pm 0.27\end{array}$ & $0.38 \pm 0.17$ & $\begin{array}{r}866 \pm 120 \\
1290 \pm 168 \\
1248 \pm 152\end{array}$ & $\begin{array}{r}863 \pm 151 \\
1326 \pm 182 \\
1261 \pm 170\end{array}$ & $964 \pm 185$ \\
\hline
\end{tabular}

pairs for accuracy $\left(0.51\right.$ vs. $\left.0.43, t_{(17)}=0.82, P<0.42\right)$ or for RT (1290 msec vs. $1326 \mathrm{msec}, t_{(17)}=1.01, P<0.33$ ). The $\mathrm{d}^{\prime}$ was calculated based on scores of hit and false alarm for each subject. The mean $\mathrm{d}^{\prime}$ was $-0.53 \pm 0.86$, which was not significantly different from chance level $\left(\mathrm{d}^{\prime}=0, t_{(17)}=-1.62, P<0.12\right)$. Although there was no behavioral priming performance in the fMRI group, given that recognition memory for associations in the behavioral group and the fMRI group $\left(\mathrm{d}^{\prime}=-0.27 \pm 0.92\right)$ was similar, we believe it is safe to conclude that there were also no group differences in associative priming. Thus, the associative priming and item priming effects were not confounded by conscious retrieval of studied word pairs, and influences of explicit associative memory on associative priming task were successfully excluded.

\section{Neuroimaging results}

\section{Item and associative priming}

During the perceptual identification task, subjects were presented with the old, recombined, and new word pairs using a backward masking paradigm. The results showed that many brain regions were activated for different word pair comparisons. Compared with new pairs, old pairs showed decreased activation in the occipital cortex, prefrontal cortex, right PHG $(11,-22$, $-5, t=-3.67$, and $27,-49,6, t=-3.55$ ), cingulate cortex, caudate, and cerebellum; recombined pairs showed decreased activation in the occipital cortex, prefrontal cortex, right basal ganglia, and bilaterally in the cerebellum. When compared with recombined pairs, old pairs showed decreased activation in the left occipital cortex, bilateral prefrontal cortex, right posterior PHG (coordinate 22, $-33,-7, t=-3.06 ; 25,-42,3, t=-2.93$ ), and the anterior cingulate (ACC).
Priming effects are generally associated with the phenomenon of repetition suppression (Wiggs and Martin 1998; GrillSpector et al. 2006), i.e., decreased hemodynamic responses in cortical regions for repeated/old stimuli compared with new stimuli. Based on this effect, we defined priming effects by conjunction analysis of word pair comparisons. The brain regions related to item priming were identified as the conjunction of significantly activated areas observed in both old $<$ new and recombined $<$ new pairs (i.e., old items $<$ new items), while the regions related to associative priming were identified as the conjunction of significantly activated areas observed in both, old $<$ new and the old $<$ recombined pairs (i.e., old associations $<$ new associations).

The conjunction analysis showed that item priming effects were present in the cuneus (medial occipital lobe), right prefrontal cortex, right caudate, and cerebellum (Table 2; Fig. 2). This finding is consistent with those of previous neuroimaging studies (e.g., Buckner et al. 1998; Koutstaal et al. 2001; Slotnick and Schacter 2006) and confirms the relationship between priming effects and repetition suppression (Wiggs and Martin 1998; GrillSpector et al. 2006).

Similar to the item priming effects, associative priming effects (Table 2; Fig. 3) were obtained in the cuneus (medial occipital lobe, BA19) and the left inferior frontal gyrus. Different from item priming, associative priming effects were also obtained in the right posterior PHG (coordinate $21,-34,-7, t=-2.96$ for recombined $<$ new; $11,-42,-5, t=-3.67$ for old $<$ new) (Fig. 4) and the ACC. That is, the activation in these two regions significantly decreased for old associations compared with new associations (i.e., old vs. recombined pairs, and old vs. new pairs). Within the right PHG, the activation was located in the posterior part, i.e., the parahippocampal cortex, following the distinction between the anterior and posterior PHG at about $y=-20$ in

Table 2. Pair contrasts for item priming and associative priming effects in the perceptual identification task

\begin{tabular}{|c|c|c|c|c|c|c|c|c|c|}
\hline \multirow[b]{2}{*}{ L/R } & Region & $\mathbf{t}$ & $x$ & $y$ & z & $\mathbf{t}$ & $x$ & $y$ & Z \\
\hline & Item priming & \multicolumn{4}{|c|}{ Old $<$ New } & \multicolumn{4}{|c|}{ Recom $<$ New } \\
\hline $\mathrm{L}$ & Occipital (BA18/19) & -3.83 & -4 & -78 & -12 & -2.75 & -3 & -85 & -10 \\
\hline $\mathrm{R}$ & Precentral/MFG & -4.16 & 27 & 3 & 27 & -4.52 & 27 & 2 & 27 \\
\hline $\mathrm{R}$ & Precentral/MFG & -3.35 & 26 & -13 & 55 & -3.95 & 26 & -13 & 56 \\
\hline $\mathrm{R}$ & SFG & -3.84 & 2 & -16 & 71 & -2.72 & 10 & -15 & 66 \\
\hline $\mathrm{L}$ & Postcentral/BA3 & -3.81 & -47 & -15 & 53 & -3.15 & -47 & -15 & 53 \\
\hline \multirow[t]{3}{*}{$\mathrm{L}$} & Precentral/BA6 & -3.19 & -24 & -19 & 68 & -3.57 & -22 & -13 & 73 \\
\hline & Precentral/BA6 & -3.50 & -40 & -26 & 59 & -2.54 & -38 & -19 & 65 \\
\hline & PCC/BA30 & -2.69 & -5 & -54 & 6 & -2.58 & -3 & -53 & 5 \\
\hline L & Cerebellum & -2.75 & 2 & -74 & -24 & -3.85 & 0 & -72 & -26 \\
\hline \multirow[t]{2}{*}{$\mathrm{R}$} & Caudate & -4.14 & 17 & 2 & 22 & -2.82 & 16 & 2 & 21 \\
\hline & Associative priming & \multicolumn{4}{|c|}{ Old < Recom } & \multicolumn{4}{|c|}{ Old $<$ New } \\
\hline $\mathrm{L}$ & Occipital (BA19) & -2.65 & -7 & -83 & 34 & -3.32 & -5 & -77 & 33 \\
\hline $\mathrm{R}$ & PHC (BA36) & -2.96 & 21 & -34 & -7 & -3.67 & 11 & -42 & -5 \\
\hline & $\mathrm{IFG} / \mathrm{BA} 44$ & -2.80 & -54 & 8 & 7 & -3.89 & -56 & 7 & 7 \\
\hline L & ACC (BA24) & -2.49 & 2 & 17 & 27 & -3.05 & 2 & 18 & 28 \\
\hline
\end{tabular}

L indicates left hemisphere; R, right hemisphere; MFG, middle frontal gyrus; SFG, superior frontal gyrus; PCC, posterior cingulate cortex; PHC, parahippocampal cortex; IFG, inferior frontal gyrus; and ACC, anterior cingulate cortex. 


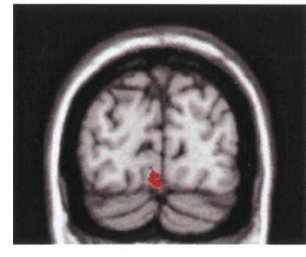

$y=-81$

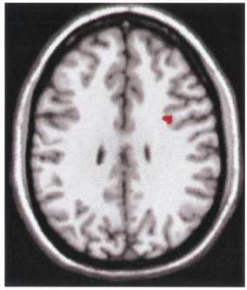

$z=29$

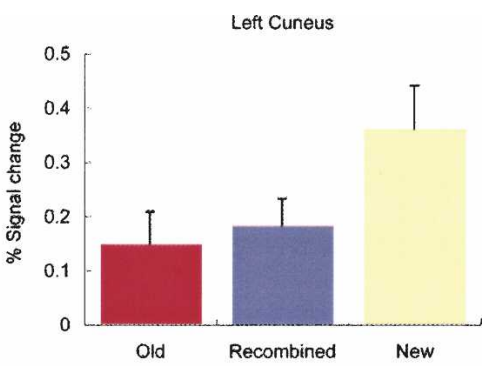

Right MFG

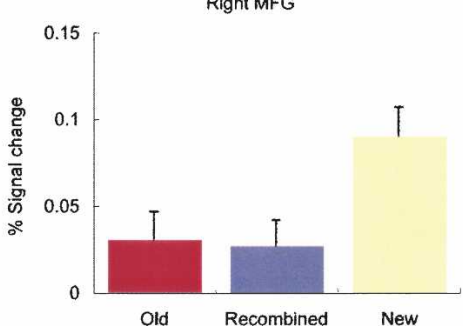

Figure 2. Brain regions showing both old $<$ new pairs and recombined $<$ new pairs (item priming effect) in the perceptual identification task. (Left) Conjunction maps overlayed on a standard anatomical structure, masked by significant omnibus experimental effect at a threshold of $P<10^{-4}$. The cuneus and the right MFG elicited decreased activation for the old and recombined pairs compared with the new pairs $(P<0.05)$. The left hemisphere is displayed on the left. (Right) Percentage of signal change (and standard errors) of different types of word pairs for regions identified in the activation maps.

Talairach space (Pruessner et al. 2002; Henson 2003). ${ }^{5}$ These data and other findings (e.g., Badgaiyan et al. 2003; Henke et al. 2003a,b; Preston and Gabrieli 2008) suggest that associative priming is different from item priming in that it is related to activation in the MTL. On the other hand, we did not find activation of the hippocampus for the contrasts between the word pairs, which suggests a functional dissociation between the hippocampus and the parahippocampal cortex in associative priming.

In addition to repetition suppression effects, several brain areas showed increased activation (repetition enhancement) for old vs. recombined, old vs. new, and recombined vs. new. For example, the prefrontal cortex was activated for old vs. recombined pairs (coordinate $-34,-8,65, t=3.57$ ) and recombined vs. new pairs (coordinate $34,17,25, t=3.33$ ). Note that the cluster that showed repetition enhancement in the fusiform/MTL was small (old vs. recombined pairs, coordinate $35,-36,-24$, $t=2.86$, EPI voxel $<1$ ). Moreover, when conjunction analysis was conducted for direct contrasts between word pairs as described before, there were no overlapping areas for the old $>$ recombined and old $>$ new effects, which suggests that repetition enhancement is not the main mechanism contributing to item and associative priming effects.

\section{Associative recognition}

As revealed by the behavioral data, recognition memory for associations was at chance level; i.e., subjects could not distinguish the old and the recombined pairs. To get a hint on whether different mechanisms are involved in implicit and explicit

${ }^{5}$ To diminish the influence of spatial variability across subjects, we also conducted a conjunction analysis for individual subjects and found a consistent pattern in the MTL for associative priming. The results showed that 12 out of 13 subjects had PHG activation for the old < new pairs; nine subjects, for old <recombined pairs; and nine subjects, for the conjunction of the above contrasts. memory, we analyzed significant BOLD signal changes for successfully recognized old vs. recombined pairs (hit vs. correct rejection [CR]), despite chance performance on the behavioral level.

The brain areas that showed hit $>C R$ and hit $<$ CR effects were in the fusiform gyrus, bilateral occipital lobe, MTL, MTG, prefrontal cortex, ACC, and parietal cortex. Within the MTL (Fig. 5 ), the right parahippocampal cortex (coordinate $23,-42,-14$, $t=-3.67 ; 40,-31,-16, t=-2.64)$, and different from associative priming, the bilateral hippocampus (coordinate $-25,-25$, $-5, t=-4.10 ; 20,-20,-6, t=-5.89)$ exhibited the hit $<\mathrm{CR}$ pattern. Note that the activation of the right parahippocampal cortex during the explicit memory task was more posterior and more lateral than that during the priming task. In addition, the left PHG/fusiform (coordinate $-35,-38,-14, t=3.54$ ) exhibited the hit $>$ CR pattern. The other two clusters in the bilateral

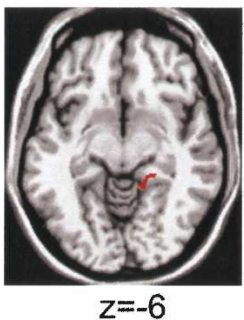

$z=-6$

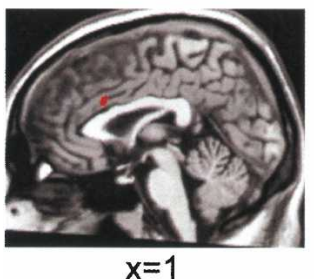

$x=1$
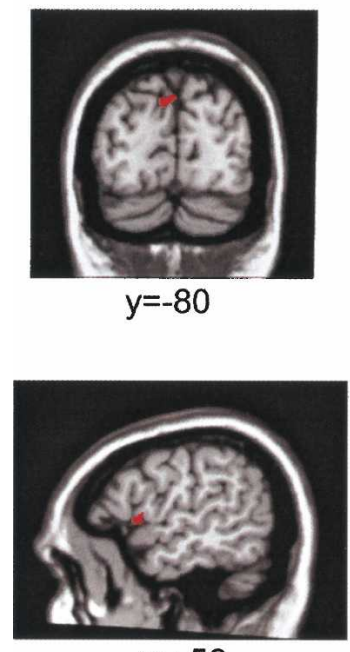

$x=-53$
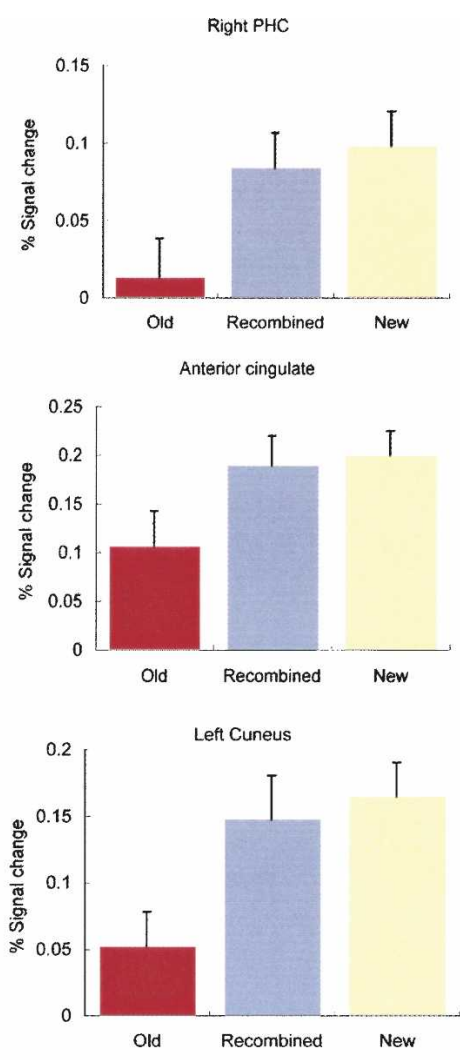

Left IFG

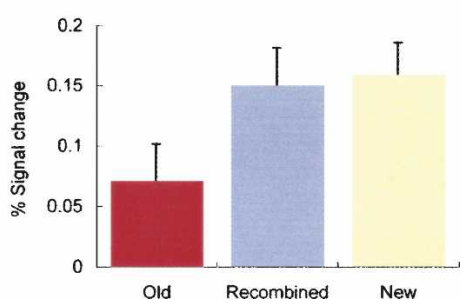

Figure 3. Brain regions showing both old $<$ new pairs and old $<$ recombined pairs (associative priming effect) in the perceptual identification task. (Left) Conjunction maps overlayed on a standard anatomical structure, masked by significant omnibus experimental effect at a threshold of $P<10^{-4}$. The right parahippocampal cortex (PHC), the anterior cingulate cortex (ACC), occipital cortex, and the left IFG elicited decreased activation for old pairs compared with recombined and new pairs $(P<0.05)$. The left hemisphere is displayed on the left. (Right) Percentage of signal change (and standard errors) of different types of word pairs for regions identified in the activation maps. 


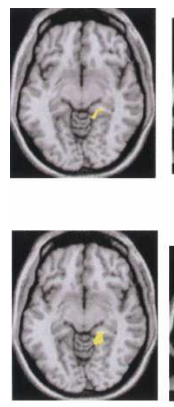

$Z=-5$
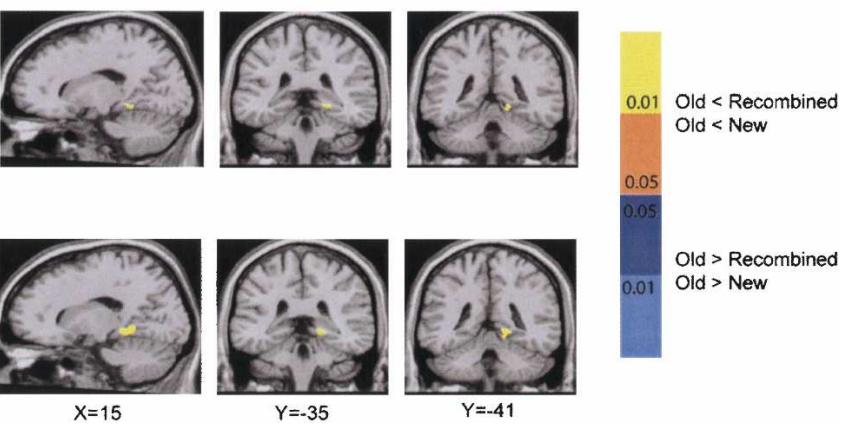

Figure 4. The activity of the right parahippocampal cortex for comparison of old vs. new pairs and old vs. recombined pairs in the perceptual identification task. The activation maps overlayed on a standard anatomical structure, masked by significant omnibus experimental effect at a threshold of $P<10^{-4}$. The right parahippocampal cortex exhibited decreased activation for the old pairs compared with recombined and new pairs $(P<0.05)$. The left hemisphere is displayed on the left.

perceptual format). The involvement of the PHG in associative priming is further supported by a variety of previous studies (e.g., Chun and Phelps 1999; Manns and Squire 2001; O'Kane et al. 2005; Preston and Gabrieli 2008). For example, damage to MTL regions outside the hippocampus is related to implicit contextual learning in amnesia compared with hippocampal lesions (Chun and Phelps 1999; Manns and Squire 2001). Using the same paradigm as Chun and Phelps (1999), Preston and Gabrieli (2008) have found that the anterior PHG is involved in contextual learning independent of explicit memory. In addition, in the latter study, the parahippocampal cortex exhibited decreased activation for repeated contexts that were later recog-

PHG exhibited the hit $>$ CR pattern (coordinate $-25,-44,-11$, $t=3.65 ; 34,-33,-11, t=3.53$ ), although they were small (1 EPI voxel) and did not reach the criteria level. These data suggest that both the bilateral hippocampus and the PHG participate in the explicit recognition task, with the parahippocampal cortex exhibiting both response patterns of hit $<$ CR (right) and hit $>C R$ (left).

\section{Discussion}

The aim of this study was to investigate whether the MTL participates in associative priming when contributions from explicit memory are controlled for. By employing behavioral and eventrelated fMRI measures in normal subjects, our study revealed three main results: First, associative priming was dissociated from associative recognition in behavioral performance; thus, explicit contamination of associative priming was excluded. Second, the right parahippocampal cortex and the ACC, together with the occipital and the prefrontal cortex, were involved in associative priming and showed decreased activation for old associations vs. new associations. Conversely, the hippocampus did not exhibit activation for item or associative priming. Third, the bilateral hippocampus and the parahippocampal cortex were activated in the associative recognition memory task. These findings indicated a functional dissociation within the MTL in that the parahippocampal cortex, but not the hippocampus, was involved in the implicit retrieval of associative memories. In addition, they provided evidence for the view that the MTL is essential for retrieving associative information, irrespective of conscious awareness.

The critical finding of our study is the functional dissociation of the MTL in the associative priming task. Consistent with our prediction, the right parahippocampal cortex (i.e., the posterior PHG) exhibited decreased activation for old associations vs. new associations, but the hippocampus did not exhibit decreased activation for the word pair contrasts. As we used a perceptual encoding task and tested the associations between words by their pair formats, the involvement of the parahippocampal cortex in associative priming may be perceptually based (i.e., the cued information matched the studied information by its nized, and this repetition suppression effect did not differ between subjects with and without explicit recognition memory. The left parahippocampal cortex is also more activated when the tasks are the same (e.g., size judgment at both study and test) than when they are different (e.g., shape judgment at study, and size judgment at test) (O'Kane et al. 2005). To the extent to which different tasks in the study and test phases can be considered as different contextual association for single items (Schacter et al. 2004), the results of the O'Kane et al. (2005) study provide support for the view that decreased activation of the parahippocampal cortex is confined to implicit retrieval of associative information.

On the other hand, although the bilateral hippocampus was not involved in the associative priming task, it participated in the associative recognition task. Even though the pattern of explicit results has to be interpreted with caution due to chance performance on recognition memory for associations, they may suggest that the parahippocampal cortex and the hippocampus serve distinct roles in different aspects of associative memory (e.g., Cabeza et al. 2001; Duzel et al. 2003; Preston and Gabrieli 2008; for reviews, see Eichenbaum 2004; Schacter et al. 2004; Squire et al. 2004). According to the model proposed by Diana et al. (2007) and Eichenbaum et al. (2007), the PHG is involved in retrieval of contextual information, whereas the hippocampus is involved in recollection and binding of items and context. In our study, only when subjects formed associations between unre-
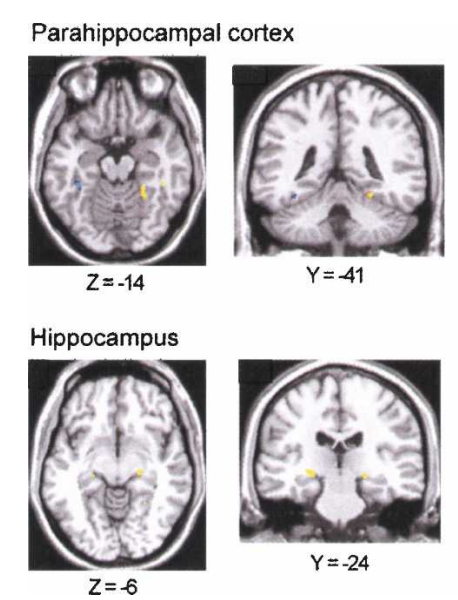

$\mathrm{Y}=-24$
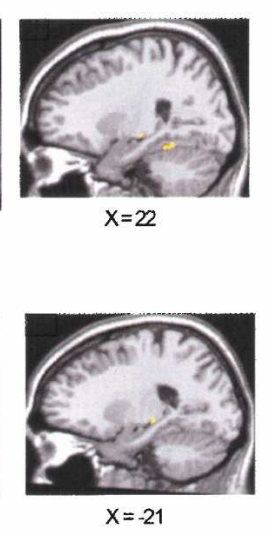

Figure 5. Brain regions showing hit vs. $C R$ in the associative recognition task. The activation maps overlayed on a standard anatomical structure, masked by significant omnibus experimental effect at a threshold of $P<10^{-4}$. The right parahippocampal cortex and the bilatera hippocampus were activated for hit $<$ correct rejection $(P<0.05)$. The left hemisphere is displayed on the left. 
lated words, did they name the old pairs more accurately than the recombined pairs. These data and others (e.g., O'Kane et al. 2005; Preston and Gabrieli 2008) provide evidence for a functional dissociation between the PHG and the hippocampus. They further extend this proposal in that the parahippocampal cortex is involved in processes that unconsciously retrieve information between unrelated items. When retrieval is accompanied by awareness, the hippocampus is additionally activated. As the hippocampus exhibited a hit $<$ CR pattern, it may play a role in the conscious detection of the novelty of the new associations in recombined pairs.

The result of a functional dissociation of MTL subregions in associative priming was obtained under testing conditions under which contamination from explicit memory was controlled for. This is an important prerequisite for determining neural correlates of associative priming given that explicit contamination was a critical confound in previous studies (e.g., Badgaiyan et al. 2003; Henke et al. 2003a,b. For reviews, see Henson 2003; Schacter et al. 2004). Our study showed that associative priming was dissociated from explicit associative recognition memory in normal subjects. Subjects had higher accuracy for old vs. recombined word pairs in the priming task, even though the pairs were presented only briefly (i.e., at their threshold duration). On the other hand, as subjects learned word pairs under perceptual encoding, their performance on associative explicit memory was at chance level.

We should note that some studies have found that the hippocampus is activated when subjects retrieve repeated association by partial cues (e.g., word stems in Badgaiyan et al. [2003]; faces in Henke et al. [2003a,b]). It raises the possibility that both excitatory and inhibitory BOLD responses are related to human MTL during memory tasks (Fried et al. 2002; Ojemann et al. 2002), especially when associative priming relies on conceptual/ spatial information (e.g., Badgaiyan et al. 2003; Henke et al. $2003 a, b)$. However, given that some of the previous studies did not obtain behavioral dissociations between associative priming and explicit tasks (e.g., Henke et al. 2003a), it is unclear whether explicit memory plays a role in associative priming in these studies. Our data suggest that the hippocampus is recruited by explicit associative memory but not by the implicit retrieval of associative information. By using paradigms to dissociate implicit and explicit memory (e.g., Schott et al. 2005; Daselaar et al. 2006), further studies may be promising to give us a more consistent picture of the mechanism by which the MTL mediates different types of associative memory.

In addition to the PHG, the ACC was also activated in the associative priming condition. Previous studies have shown that the ACC has many different functions; one is to participate in conflict monitoring (Carter et al. 1998). In the domain of memory retrieval, the ACC shows stronger activation when subjects had to discriminate old words from semantically related new words (von Zerssen et al. 2001). As this activation pattern was also present for erroneous "old" responses in the latter study, it is reasonable to assume that the ACC is related to performance monitoring under conditions of high task difficulty or high conflicting. In the present study, the recombined pair changed the association between the two words that were initially studied together. By this, it is possible that the memory for old pairings interfered with the reading of recombined word pairs and that this may have increased processing conflict and monitoring demands. Moreover, as subjects could not distinguish old and recombined pairs, this monitoring may be unconscious in nature. Therefore, our data are more consistent with the view that ACC activity is not necessarily related to awareness (e.g., Critchley et al. 2005; Hester et al. 2005) than the view that the ACC is crucial for conscious conflict control (Dehaene et al. 2003).
In conclusion, by controlling explicit contamination, our study showed a functional dissociation within the MTL memory system in that the right PHG but not the hippocampus was involved in associative priming. The PHG exhibited decreased activation for old pairs compared with both recombined and new pairs. On the other hand, the bilateral hippocampus and the PHG were involved in explicit associative memory. These data provide evidence that different regions within the MTL play distinct roles in explicit and implicit associative memory.

\section{Materials and Methods}

\section{Subjects}

Thirty-three healthy, right-handed subjects (18 male, 15 female) participated in this study, with a mean age $21.58 \pm 2.04$ yr. Fifteen (eight male) of them participated in the fMRI group. Given that speaking aloud will produce excessive head movement, subjects were asked to read pairs silently in the MR scanner. Two subjects' fMRI data were excluded because of large head motion. A separate behavioral group (18 subjects, 10 male) was selected to perform the same tasks outside of the scanner with loud speaking as the response. There were no significant gender and age differences between the two groups. All subjects were paid for their participation and gave written informed consent in accordance with procedures and protocols approved by the Institutional Review Board of Beijing MRI Center for Brain Research.

\section{Stimuli}

A total of 320 two-character Chinese concrete words were selected to form 160 unrelated word pairs; for example, 玫瑰一磁铁 (rose-magnet), 钥题一核桃 (key-walnut). No obvious semantic, orthographic, or phonological relationship existed between two words in each pair. All context and target words had medium frequencies of occurrence $(187 \pm 90$ per million) and medium numbers of stroke $(17.51 \pm 4.56)$. Since all Chinese characters correspond to one spoken syllable, each word (two characters) has two syllables.

The word pairs were divided into two sets, i.e., a threshold set and a test set. The threshold set (90 word pairs) was used to determine the exposure duration for each subject. The test set $(60$ word pairs) was used in both the behavioral and the fMRI groups. It was further divided into three subsets, each composed of 20 pairs that were used as repeated, recombined, and new pairs, respectively. The repeated and recombined subsets were presented in both study and test sessions, and the new subset was used as the baseline in the perceptual identification test. Each word and its characters appeared only once in all three subsets. Word frequency $\left(F_{(1,298)}=0.36, P<0.59\right)$ and stroke $\left(F_{(1,298)}=0.23, P<0.63\right)$ were not significantly different between the threshold set and the test set, or among the three subsets $\left(F_{(2,117)}=0.02\right.$ for frequency, and $F_{(2,117)}=0.19$ for stroke, respectively). The arrangement of the three subsets was counterbalanced so that each word pair had an equal chance to be repeated, recombined, and new pairs across subjects. The remaining 10 pairs were used for practice in both groups.

\section{Procedure}

The study included two stages. In the first stage, the optimal exposure durations for minimizing possible explicit memory contamination were determined for each subject. A series of tests, each with 10 unrelated word pairs in the threshold set, were carried out outside of the scanner. Before the presentation of each word pair, a fixation "+" was displayed in the center of the screen. Five hundred milliseconds later, a word pair was presented for a certain duration and then masked immediately by an irregular and meaningless drawing consisting of character-like strokes. Subjects were asked to speak out the word pairs as quickly and accurately as possible. The exposure durations of the word pairs changed according to the subjects' performance. If the percentage of correct report was higher than $30 \%$, the exposure du- 
ration of the next 10 pairs was reduced by $16.7 \mathrm{msec}$. Otherwise, if the percentage correct was lower than $20 \%$, the duration of the next 10 pairs was increased by $16.7 \mathrm{msec}$. By use of this procedure, the exposure duration for each subject was gradually adjusted to yield a correct identification rate of $20 \%-40 \%$. For more details on the threshold procedure, see the study of Yang et al. (2003).

The second stage, either conducted in the scanner for the fMRI group or outside of the scanner for the behavioral group, was composed of three tasks, i.e., a perceptual encoding task, a perceptual identification (priming) task, and an explicit recognition task. During the encoding task, subjects were asked to decide whether the two Chinese words had the same structural configurations (e.g., up-down, left-right), given each Chinese character has distinct orthographical structure. Each pair was presented for 2 sec and then replaced by "+" on the screen. During the perceptual identification task, subjects were asked to covertly read the quickly flashed word pairs. Sixty test pairs, consisting of repeated, recombined, and new pairs, mixed with 20 masks were presented in random order with the individually predetermined exposure duration and then masked with the character-like strokes. During the explicit recognition task, subjects were asked to make old/ recombined judgment as quickly and accurately as possible. Each word pair was presented for 2 sec in either the same or different combinations as those in the study session. To control for rehearsal of the studied stimuli, subjects performed a mental arithmetic task (serial subtraction of 7 starting from 1000) continuously for 3 min between the two tasks.

In order to get behavioral priming scores, the behavioral group followed the same procedure except that subjects overtly read the quickly flashed word pairs in the perceptual identification task. For the behavioral group, the accuracy data were recorded for all 18 subjects, and RTs were recorded additionally for eight subjects. For the fMRI group, a rapid event-related design was applied in both the perceptual identification and recognition sessions, with 268 and 147 volumes collected, respectively. All stimuli, including word pairs and the fixations, were optimally intermixed in a pseudo-random order.

\section{MRI acquisition}

MR data were collected on a Siemens 3 Tesla scanner (Magnetom Trio). Stimuli were presented through a back projector. Subjects viewed the stimuli through a mirror attached to the head coil. The anatomical data were acquired using high-resolution SPGR $(\mathrm{TR}=845 \mathrm{msec}, \mathrm{FOV}=22 \mathrm{~cm}$, matrix $=256 \times 256$, resolution $=1 \times 1 \times 1.3 \mathrm{~mm}^{3}$ ) sequence before the study session. The whole brain functional data were acquired using a gradient echo, echo-planar imaging (EPI) sequence $(\mathrm{TR}=2 \mathrm{sec}, \mathrm{TE}=30 \mathrm{msec}$, flip angle $=90^{\circ}$, FOV $=22 \mathrm{~cm}$, matrix $=64 \times 64$, resolution $=3.75 \times 3.75 \times 5 \mathrm{~mm}^{3}$ ). To better observe the signals in the MTL regions, oblique slices that were perpendicular to the long axis of the hippocampus were adopted.

\section{Data analysis}

AFNI (http://afni.nimh.nih.gov) was used for fMRI data preprocessing and statistical analyses. The first five EPI volumes before magnetization and intensity which remained steady state were discarded. The remaining volumes were registered to the nearest volume close to anatomical images to correct subjects' head movement. Spatial smooth with a root-mean-square (RMS) width of $4 \mathrm{~mm}$ was applied to all registered volumes. To better compare data between subjects, all MRI signals in each volume were normalized to the percentage of signal change before performing the deconvolution analysis. In 3dDeconvolve, a time window of six TRs $(12 \mathrm{sec})$ was selected to model the hemodynamic response of each stimulus. Altogether 18 regressors of interest (repeated, recombined, and old pairs) and six regressors of noninterest motion parameters were applied in the perceptual identification session, and the $\beta$ weights of the impulse response function (IRF) were used to estimate the amplitude for each condition (vs. fixation).

After transforming functional IRF of each subject to stan- dard stereotactic coordinates of the Talairach and Tournoux atlas (Talairach and Tournoux 1988), a voxel-wised mixed-effect model of ANOVA was adopted in the group analysis, with word pair type (old, recombined, old) as the fixed factor for the perceptual identification task. The conjunction analysis was then applied to find the activated brain areas for item and associative priming effects. The group activation map was first masked by significant omnibus experimental effect at a threshold of $P<10^{-4}$ and then masked by significant pair contrasts at the threshold of $P<0.05$ (two-tailed, above 140 voxels). We defined the priming effects in terms of repetition suppression (Wiggs and Martin 1998; Grill-Spector et al. 2006), i.e., decreased hemodynamic responses in cortical regions for repeated (old) stimuli compared with new stimuli. During the perceptual identification task, old pairs included the repetition of identical pairs from the study session; recombined pairs were repeated (old) items in new associations, whereas new pairs had both new items and new associations. Therefore, if brain regions showed decreased activation for both old vs. new pairs and recombined vs. new pairs, they were considered to reflect item priming effects; i.e., old items showed more repetition suppression effects. Similarly, if brain regions showed decreased activation for both old vs. recombined pairs and old vs. new pairs, they were considered to reflect associative priming effects; i.e., old association showed repetition suppression effects compared with new associations. To demonstrate the mean percentage of signal changes in regions related to item and associative priming, the $\beta$ weights were averaged across subjects. For the explicit recognition task, the difference between correct old (hit) and correct recombined (correct rejection, CR) was compared to find the association-related brain activation $(P<0.05$, two-tailed, above 140 voxels), after the group activation map was masked by the significant omnibus experimental effect at a threshold of $P<10^{-4}$.

\section{Acknowledgments}

We thank Dr. Gang Chen from the National Institutes of Health for his invaluable help on data analysis. This research was supported by grants from the Foundation for the Author of National Excellent Doctoral Dissertation of China (J.Y., 200207), Global Research Initiative Program, National Institutes of Health (J.Y., 2008) and Chinese Ministry of Science and Technology (X.W., 2007CB512300).

\section{References}

Aggleton, J.P. and Brown, M.W. 2006. Interleaving brain systems for episodic and recognition memory. Trends Cogn. Sci. 10: 455-463.

Badgaiyan, R.D., Schacter, D.L., and Alpert, N.M. 1999. Auditory priming within and across modalities: Evidence from positron emission tomography. J. Cogn. Neurosci. 11: 337-348.

Badgaiyan, R.D., Schacter, D.L., and Alpert, N.M. 2001. Priming within and across modalities: Exploring the nature of rCBF increases and decreases. Neuroimage 13: 272-282.

Badgaiyan, R.D., Schacter, D.L., and Alpert, N.M. 2003. Priming of new associations: A PET study. Neuroreport 14: 2475-2479.

Buckner, R.L. and Wheeler, M.E. 2001. The cognitive neuroscience of remembering. Nat. Rev. Neurosci. 2: 624-634.

Buckner, R.L., Goodman, J., Burock, M., Rotte, M., Koutstaal, W., Schacter, D., Rosen, B., and Dale, A.M. 1998. Functional-anatomic correlates of object priming in humans revealed by rapid presentation event-related fMRI. Neuron 20: 285-296.

Cabeza, R., Rao, S.M., Wagner, A.D., Mayer, A.R., and Schacter, D.L. 2001. Can medial temporal lobe regions distinguish true from false? An event-related functional MRI study of veridical and illusory recognition memory. Proc. Natl. Acad. Sci. 98: 4805-4810.

Carter, C.S., Braver, T.S., Barch, D.M., Botvinick, M.M., Noll, D., and Cohen, J.D. 1998. Anterior cingulate cortex, error detection, and the online monitoring of performance. Science 280: 747-749.

Chun, M.M. and Phelps, E.A. 1999. Memory deficits for implicit contextual information in amnesic subjects with hippocampal damage. Nat. Neurosci. 2: 844-847.

Critchley, H.D., Tang, J., Glaser, D., Butterworth, B., and Dolan, R.J. 2005. Anterior cingulate activity during error and autonomic response. Neuroimage 27: 885-895.

Daselaar, S.M., Fleck, M.S., Prince, S.E., and Cabeza, R. 2006. The medical temporal lobe distinguishes old from new independently of 
consciousness. J. Neurosci. 26: 5835-5839.

Davachi, L. 2006. Item, context and relational episodic encoding in humans. Curr. Opin. Neurobiol. 16: 693-700.

Dehaene, S., Artiges, E., Naccache, L., Martelli, C., Viard, A., Schurhoff, F., Recasens, C., Martinot, M.L.P., Leboyer, M., and Martinot, J.L. 2003. Conscious and subliminal conflicts in normal subjects and patients with schizophrenia: The role of the anterior cingulate. Proc. Natl. Acad. Sci. 100: 13722-13727.

Diana, R.A., Yonelinas, A.P., and Ranganath, C. 2007. Imaging recollection and familiarity in the medial temporal lobe: A three-component model. Trends Cogn. Sci. 11: 379-386.

Duzel, E., Habib, R., Rotte, M., Guderian, S., Tulving, E., and Heinze, H.J. 2003. Human hippocampal and parahippocampal activity during visual associative recognition memory for spatial and nonspatial stimulus configurations. J. Neurosci. 23: 9439-9444.

Eichenbaum, H. 2004. Hippocampus: Cognitive processes and neural representations that underlie declarative memory. Neuron 44: $109-120$.

Eichenbaum, H., Yonelinas, A.P., and Ranganath, C. 2007. The medial temporal lobe and recognition memory. Annu. Rev. Neurosci. 30: $123-152$.

Fried, I., Cameron, K.A., Yashar, S., Fong, R., and Morrow, J.W. 2002. Inhibitory and excitatory responses of single neurons in the human medial temporal lobe during recognition of faces and objects. Cereb. Cortex 12: $575-584$.

Gabrieli, J.D.E., Keane, M.M., Zarella, M.M., and Poldrack, R.A. 1997. Preservation of implicit memory for new associations in global amnesia. Psychol. Sci. 8: 326-329.

Gazzaniga, M.S., Ivry, R.B., and Mangun, G.R. 2002. Cognitive neuroscience: The biology of the mind, 2nd ed. W.W. Norton, New York.

Goshen-Gottstein, Y. and Moscovitch, M. 1995. Repetition priming effects for newly formed associations are perceptually based: Evidence from shallow encoding and format specificity. J. Exp. Psychol. Learn. Mem. Cogn. 21: 1249-1262.

Goshen-Gottstein, Y., Moscovitch, M., and Melo, B. 2000. Intact implicit memory for newly formed verbal associations in amnesic patients following single study trials. Neuropsychology 14: 570-578.

Graf, P. and Schacter, D.L. 1985. Implicit and explicit memory for new associations in normal and amnesic subjects. J. Exp. Psychol. Learn. Mem. Cogn. 11: 501-518.

Grill-Spector, K., Henson, R., and Martin, A. 2006. Repetition and the brain: Neural models of stimulus-specific effects. Trends Cogn. Sci. 10: $14-23$.

Henke, K., Mondadori, C.R.A., Treyer, V., Nitsch, R.M., Buck, A., and Hock, C. 2003a. Nonconscious formation and reactivation of semantic associations by way of the medial temporal lobe. Neuropsychologia 41: 863-876.

Henke, K., Treyer, V., Nagy, E.T., Kneifel, S., Dursteler, M., Nitsch, R.M., and Buck, A. 2003b. Active hippocampus during nonconscious memories. Conscious. Cogn. 12: 31-48.

Henson, R.N.A. 2003. Neuroimaging studies of priming. Prog. Neurobiol. 70: $53-81$.

Henson, R. 2005. A mini-review of fMRI studies of human medial temporal lobe activity associated with recognition memory. Q J Exp Psychol B 58: 340-360.

Hester, R., Foxe, J.J., Molholm, S., Shpaner, M., and Garavan, H. 2005. Neural mechanisms involved in error processing: A comparison of errors made with and without awareness. Neuroimage 27: 602-608.

Koutstaal, W., Verfaellie, M., and Schacter, D.L. 2001. Recognizing identical versus similar categorically related common objects: Further evidence for degraded gist representations in amnesia. Neuropsychology 15: 268-289.

Manns, J.R. and Squire, L.R. 2001. Perceptual learning, awareness, and the hippocampus. Hippocampus 11: 776-782.

Martin, A. and Gotts, S.J. 2005. Making the causal link: Frontal cortex activity and repetition priming. Nat. Neurosci. 8: 1134-1135.

Mayes, A.R. and Gooding, P. 1989. Enhancement of word completion priming in amnesiacs by cueing with previously novel associates.
Neuropsychologia 27: 1057-1072.

Mayes, A.R., Holdstock, J.S., Isaac, C.L., Montaldi, D., Grigor, J., Gummer, A., Cariga, P., Downes, J.J., Tsivilis, D., Gaffan, D., et al. 2004. Associative recognition in a patient with selective hippocampal lesions and relatively normal item recognition. Hippocampus 14: 763-784.

Mayes, A., Montaldi, D., and Migo, E. 2007. Associative memory and the medial temporal lobes. Trends Cogn. Sci. 11: 126-135.

Moscovitch, M., Winocur, G., and McLachlan, D. 1986. Memory as assessed by recognition and reading time in normal and memory-impaired people with Alzheimer's-disease and other neurological disorders. J. Exp. Psychol. Gen. 115: 331-347.

Musen, G. and Squire, L.R. 1993. Implicit learning of color-word associations using a stroop paradigm. J. Exp. Psychol. Learn. Mem. Cogn. 19: 789-798.

Ojemann, G.A., Schoenfield-McNeill, J., and Corina, D.P. 2002. Anatomic subdivisions in human temporal cortical neuronal activity related to recent verbal memory. Nat. Neurosci. 5: 64-71.

O'Kane, G., Insler, R.Z., and Wagner, A.D. 2005. Conceptual and perceptual novelty effects in human medial temporal cortex. Hippocampus 15: 326-332.

Preston, A.R. and Gabrieli, J.D.E. 2008. Dissociation between explicit memory and configural memory in the human medial temporal lobe. Cereb. Cortex (in press). doi: 10.1093/cercor/bhm245.

Pruessner, J.C., Kohler, S., Crane, J., Pruessner, M., Lord, C., Byrne, A., Kabani, N., Collins, D.L., and Evans, A.C. 2002. Volumetry of temporopolar, perirhinal, entorhinal and parahippocampal cortex from high-resolution MR images: Considering the variability of the collateral sulcus. Cereb. Cortex 12: 1342-1353.

Schacter, D.L., Dobbins, I.G., and Schnyer, D.M. 2004. Specificity of priming: A cognitive neuroscience perspective. Nat. Rev. Neurosci. 5: $853-862$.

Schott, B.H., Henson, R.N., Richardson-Klavehn, A., Becker, C., Thoma, V., Heinze, H.J., and Duzel, E. 2005. Redefining implicit and explicit memory: The functional neuroanatomy of priming, remembering, and control of retrieval. Proc. Natl. Acad. Sci. 102: 1257-1262.

Shimamura, A.P. and Squire, L.R. 1989. Impaired priming of new associations in amnesia. J. Exp. Psychol. Learn. Mem. Cogn. 15: $721-728$.

Slotnick, S.D. and Schacter, D.L. 2006. The nature of memory related activity in early visual areas. Neuropsychologia 44: 2874-2886.

Squire, L.R., Ojemann, J.G., Miezin, F.M., Petersen, S.E., Videen, T.O., and Raichle, M.E. 1992. Activation of the hippocampus in normal humans: A functional anatomical study of memory. Proc. Natl. Acad. Sci. 89: 1837-1841.

Squire, L.R., Stark, C.E.L., and Clark, R.E. 2004. The medial temporal lobe. Annu. Rev. Neurosci. 27: 279-306.

Talairach, J. and Tournoux, P. 1988. Co-planar stereotaxic atlas of the human brain. 3-dimensional proportional system: An approach to cerebral imaging. Thieme, New York.

Verfaellie, M., Martin, E., Page, K., Parks, E., and Keane, M.M. 2006. Implicit memory for novel conceptual associations in amnesia. Cogn. Affect. Behav. Neurosci. 6: 91-101.

von Zerssen, G.C., Mecklinger, A., Opitz, B., and von Cramon, D.Y. 2001. Conscious recollection and illusory recognition: An event-related fMRI study. Eur. J. Neurosci. 13: 2148-2156.

Wiggs, C.L. and Martin, A. 1998. Properties and mechanisms of perceptual priming. Curr. Opin. Neurobiol. 8: 227-233.

Yang, J.J., Weng, X.C., Guan, L.C., and Kuang, P.Z. 1999. Effects of level of processing and word type on forming memory for new associations. Acta Psychological Sinica 31: 257-265.

Yang, J.J., Weng, X.C., Guan, L.C., Kuang, P.Z., Zhang, M.Z., Sun, W.J., $\mathrm{Yu}$, S.Y., and Patterson, K. 2003. Involvement of the medial temporal lobe in priming for new associations. Neuropsychologia 41: $818-829$.

Received January 8, 2008; accepted in revised form July 5, 2008. 


\section{Erratum}

Learning \& Memory 15: 703-710 (2008)

Decreased parahippocampal activity in associative priming: Evidence from an event-related fMRI study

Jiongjiong Yang, Axel Meckingler, Mingwei Xu, Yanbing Zhao, and Xuchu Weng

Coauthor Axel Mecklinger's surname was misspelled in the author line (above) and should have printed as Mecklinger. We apologize for any confusion this may have caused. 


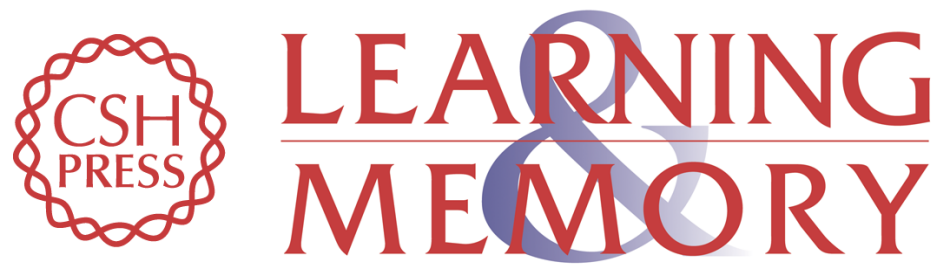

\section{Decreased parahippocampal activity in associative priming: Evidence from an event-related fMRI study}

Jiongjiong Yang, Axel Meckingler, Mingwei Xu, et al.

Learn. Mem. 2008, 15:

Access the most recent version at doi:10.1101//m.900108

Related Content

References

Email Alerting Service

\section{License}

Erratum

Learn. Mem. November , 2008 15: 856

This article cites 51 articles, 7 of which can be accessed free at:

http://learnmem.cshlp.org/content/15/9/703.full.html\#ref-list-1

Articles cited in:

http://learnmem.cshlp.org/content/15/9/703.full.html\#related-urls

Receive free email alerts when new articles cite this article - sign up in the box at the top right corner of the article or click here. 